\title{
effet d'échelle géométrique dans les milieux granulaires
}

\author{
par \\ G. Auvinet, D. Bouvard \\ Instituto de Ingenieria-Universidad Nacional de México - Mexique
}

\section{Introduction}

Les caractéristiques physiques usuelles des sols granulaires ne sont définies clairement que dans des volumes de grande dimension. Ainsi, la porosité et la granulométrie semblent perdre leur sens au niveau des particules, où elles présentent de fortes variations. II s'agit d'un effet d'échelle géométrique dont on doit tenir compte dans les applications, chaque fois que la dimension des grains est relativement grande par rapport au volume de sol considéré.

Au moyen de la théorie des fonctions aléatoires, il a été possible d'étudier ce phénomène et, en particulier, d'évaluer la dispersion des principales caractéristiques physiques des sols en fonction du volume dans lequel elles sont mesurées.

A l'aide d'un modèle de simulation numérique de la structure des sols, on a pu obtenir sur ce point certains résultats directement applicables aux problèmes pratiques de géotechnique.

\section{Représentation d'un milieu granulaire à l'aide de fonctions aléatoires}

L'arrangement des particules et des pores d'un milieu granulaire homogène peut être décrit par une fonction aléatoire spatiale $K(\bar{x})$, (Matheron, 1967) définie en tout point du milieu $\bar{x}$ comme :

$$
\begin{array}{ll}
K(\bar{x})=1 & \text { si } \bar{x} \text { est dans un pore } \\
K(\bar{x})=0 & \text { si } \bar{x} \text { est dans un grain. }
\end{array}
$$

En accord avec l'hypothèse d'homogénéité, l'espérance mathématique et la variance de cette fonction sont constantes dans tout le milieu :

$$
\begin{aligned}
E\{k(\bar{x})\} & =n \\
\operatorname{var}[K(\bar{x})] & =n(1-n)
\end{aligned}
$$

oủ $n$ est la porosité du milieu.
Par ailleurs, cette fonction aléatoire peut être considérèe comme stationnaire et ergodique suivant une direction donnée, même pour les milieux anisotropes. Son autocorrélation, son autocovariance, ainsi que son coefficient d'autocorrélation dépendent donc seulement de la différence $\bar{h}=\bar{x}_{1}-\bar{x}_{2}$ :

$$
\begin{aligned}
R\left(\bar{x}_{1}, \bar{x}_{2}\right) & =E\left\{K\left(\bar{x}_{1}\right) K\left(\bar{x}_{2}\right)\right\}=R\left(\bar{h}^{2}\right) \\
C\left(\bar{x}_{1}, \bar{x}_{2}\right) & =E\left\{\left[K\left(\bar{x}_{1}\right)-n\right]\left[K\left(\bar{x}_{2}\right)-n\right]\right\} \\
& =R(\bar{h})-n^{2}=C(\bar{h}) \\
\rho\left(\bar{x}_{1}, \bar{x}_{2}\right) & =C\left(\bar{x}_{1}, \bar{x}_{2}\right) /[n(1-n)]=\rho(\bar{h}) .
\end{aligned}
$$

L'évaluation des paramètres de la fonction $K(\bar{x})$ peut être faite expérimentalement en analysant, par exemple, des coupes d'échantillons stabilisés à la résine. Cette technique assez laborieuse est devenue plus accessible à la suite du développement de méthodes efficaces d'analyse d'images (Peralta, 1982). Pour cette étude, on a toutefois préféré utiliser la simulation numérique de la structure des milieux granulaire à l'aide d'un modèle décrit dans une publication antérieure (Auvinet, 1977).

\section{Simulation numérique d'un milieu granulaire}

\subsection{Structures simulées}

Le modèle numérique utilisé permet de mettre en place des particules sphériques dans un moule cubique d'arête a. On peut ainsi obtenir des échantillons de diverses distributions granulométriques et compacités. Le nombre de particules est seulement limité par le temps de calcul disponible. Sur le tableau I, on a présenté les caractéristiques des structures qui ont été simulées pour cette étude. On a indiqué dans chaque cas le nombre de particules, les diamètres maximum $D_{\max }$ et minimum $D_{\min }$ rapportés aux dimensions du moule, ainsi que le coefficient d'uniformité $\mathrm{C}_{\mathrm{u}}$. 


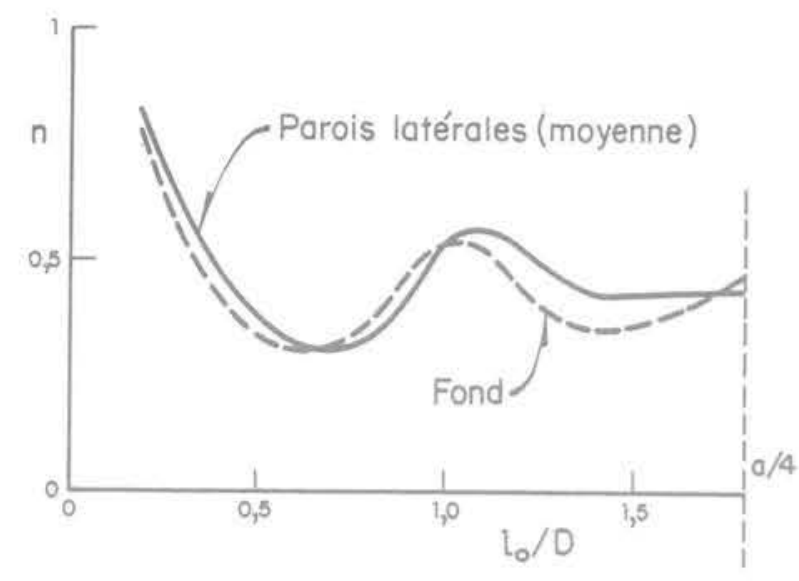

Fig. 1 Effet de paroi (structure A)

Tableau I

Caractéristiques des structures simulées

\begin{tabular}{c|c|c|c|c}
\hline Structure & $\begin{array}{c}\text { Nombre de } \\
\text { particules }\end{array}$ & $\mathrm{D}_{\min } / \mathrm{a}$ & $\mathrm{D}_{\max } / \mathrm{a}$ & $\mathrm{C}_{u}$ \\
\hline $\mathrm{A}$ & 460 & 0,14 & 0,14 & 1 \\
\hline $\mathrm{B}$ & 480 & 0,08 & 0,20 & 1,54 \\
\hline $\mathrm{C}_{1}$ & 500 & 0,04 & 0,40 & 2,56 \\
\hline $\mathrm{C}_{2}$ & 520 & 0,04 & 0,40 & 2,56 \\
\hline $\mathrm{D}$ & 1580 & 0,03 & 0,90 & 4,72 \\
\hline $\mathrm{E}$ & 700 & 0,04 & 0,40 & 2,91 \\
\hline
\end{tabular}

\subsection{Effet de paroi}

On sait que, dans un moule, la porosité présente des variations importantes au voisinage des parois. Cet effet a dû être étudié afin de définir au sein des échantillons simulés une zone de caractéristiques homogènes.

La porosité a été calculée dans une série de tranches d'échantillon de 0,025 a d'épaisseur, parallèles aux parois et 'situées à différentes distances $l_{0}$ de celles-ci. Pour les matériaux à granulométrie uniforme (fig. 1), on observe que la porosité présente une valeur maximum près de la paroi, suivie d'oscillations, qui disparaissent à une distance de l'ordre de deux diamètres de particule. Pour les matériaux à granulométrie plus variée (fig. 2), on observe à nouveau un maximum, suivi cette fois d'une réduction progressive de la porosité qui se stabilise à une distance de la parol de l'ordre de $4 \mathrm{D}_{\min }$. Pour les granulométries étudiées, cette distance ne semble pas être très sensible à la valeur du diamètre maximum $D_{\max }$. $A$ partir de ces résultats, on a pu définir dans les échantillons un noyau central cubique d'arête $0,6 \mathrm{a}$, où l'on peut considérer que l'effet de paroi est négligeable, pour toutes les granulométries simulées.

\subsection{Estimation des paramètres statistiques}

Dans le noyau de chacune des structures, il a été possible de procéder à une estimation statistique des paramètres de la fonction aléatoire $K(\bar{x})$. L'espérance

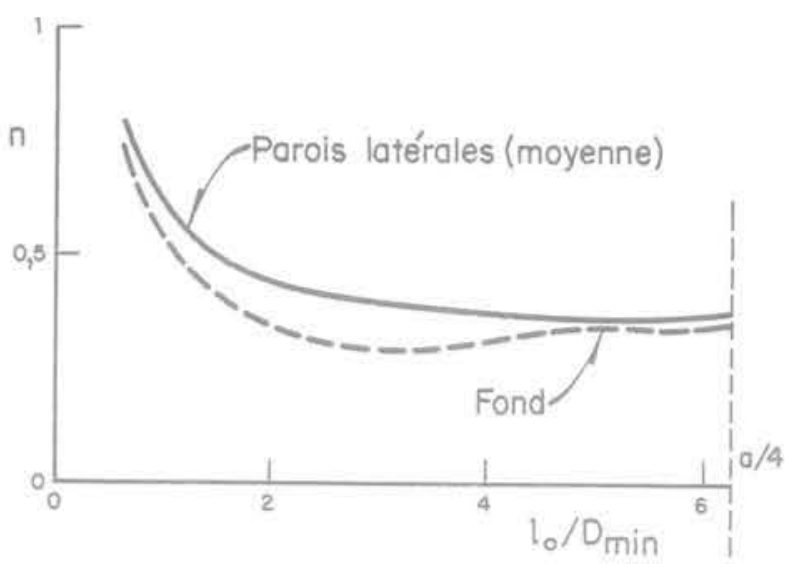

Fig. 2 Effet de paroi (structures $C_{1}$ et $C_{2}$ )

mathématique (porosité) a été évaluée par la technique de Monte-Carlo, c'est-à-dire en déterminant la fréquence avec laquelle un point placé au hasard dans l'échantillon se trouve dans les pores (Tableau II). Par ailleurs, pour estimer la fonction d'autocorrélation, on a calculé, au long de segments parallèles à une direction donnée, la moyenne spatiale suivante :

$$
R_{L, T}(h)=\frac{1}{L} \int_{0}^{L} K(\bar{x}+h i) K(\bar{x}) d \bar{x}
$$

ou : L est la longueur du segment;

í est un vecteur unitaire parallèle à la direction considérée:

$h$ est un scalaire (distance entre deux points du milieu).

Tableau /I

Estimation de la porosité $\mathrm{E}\{\mathrm{K}(\bar{x})\}$

\begin{tabular}{cc}
\hline Structure & Estimation \\
\hline A & 0,43 \\
B & 0,41 \\
C $_{1}$ & 0,42 \\
C $_{2}$ & 0,38 \\
D & 0,40 \\
E & 0,34 \\
\hline
\end{tabular}

Les résultats se sont avérés peu sensibles à la direction considérée : la fonction d'autocorrélation est pratiquement isotrope. Sur la figure 3 , on a représenté la variation du coefficient d'autocorrélation en fonction de la distance $h$, rapportée au diamètre minimum des particules.

\section{Effet d'échelle géométrique}

\subsection{Porosité}

Dans un milieu granulaire homogène, ja porosité $n_{v}$ associée à un volume donné $v$ est une variable aléatoire dont la variance dépend de la dimension du volume. Sur la figure 4 on a représenté les valeurs de la porosité mésurées dans des volumes sphériques de diamètre variable $D_{v}$, pour chacune des structures simulées. On observe les fortes variations que présente $n_{v}$ dans de petits volumes avant de tendre, 


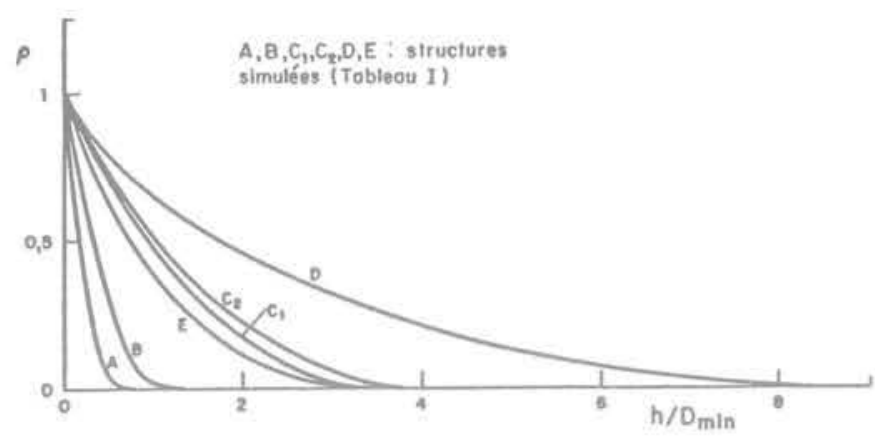

Fig. 3 Coefficient d'autocorrélation

quand $D_{v}$ augmente, vers la valeur de la porosité en masse indéfinie $n$.

La porosité associée à un volume donné peut être exprimée en fonction de $\mathrm{K}(\overline{\mathrm{x}})$, au moyen de l'intégrale stochastique suivante :

On a donc :

$$
n_{v}=\frac{1}{v} \int_{v} K(\bar{x}) d \bar{x}
$$

$$
E\left\{n_{v}\right\}=\frac{1}{v} \int_{v} E\{K(\bar{x})\} d \bar{x}=n
$$

et $\quad \operatorname{var}\left[n_{v}\right]=\sigma_{n_{v}}^{2}=\frac{1}{v^{2}} \int_{v} \int_{v} C\left(\bar{x}, \bar{x}^{\prime}\right) d \bar{x} d \bar{x}^{\prime}$

En utilisant cette dernière équation et les résultats de la figure 3 , on a pu estimer pour chacune des structures la variance théorique de la porosité associée à des volumes sphériques de différents diamètres $D_{v}$. La variation de l'écart-type $\sigma_{n_{v}}$ en fonction du rapport $D_{v} / D_{\min }$ est présentée sur la figure 5 . On voit que la dispersion décroît plus rapidement pour les matériaux denses et à granulométrie serrèe que pour les matériaux plus lâches et à granulométrie variée. L'effet de la granulométrie est le plus important.

Sur la figure 6 , les mêmes résultats sont présentés avec, en abscisses, la relation $D_{v} / D_{50}$, oủ $D_{50}$ est le diamètre des particules correspondant à un tamisat de $50 \%$. Les courbes se regroupent et, pour une valeur de $D_{v} / D_{50}$ donnée, il semble que l'écart-type ne dépende plus que de la compacité.

II est possible de vérifier que, sauf pour les plus petites valeurs de $D_{\mathrm{v}} / D_{50}$, la forme mathématique des courbes de la figure 6 est la suivante :

$$
\sigma_{n_{v}}=k\left(D_{v} / D_{50}\right)^{-3 / 2}
$$

oủ $k$ est un coefficient de l'ordre de 0,18 pour les structures les moins denses $(n=0,43)$ et de 0,11 pour les plus compactes $(n=0,34)$. L'écart-type de la porosité est donc inversement proportionnel à la racine carrée du volume de l'échantillon. Ce résultat était prévisible. En effet, on peut considérer un échantillon de volume $v$ comme la réunion de $\mathrm{N}$ échantillons plus petits, sensiblement indépendants entre eux. On sait que l'écart-type de la porosité de l'échantillon le plus grand est alors égal à celui de la porosité des plus petits, divisé par la racine carrée de $N$. C'est ce résultat que l'on retrouve dans l'équation 11, sauf pour les plus petites valeurs de $D_{v} / D_{50}$, pour lesquelles l'hypothèse d'indépendance cesse d'être acceptable.

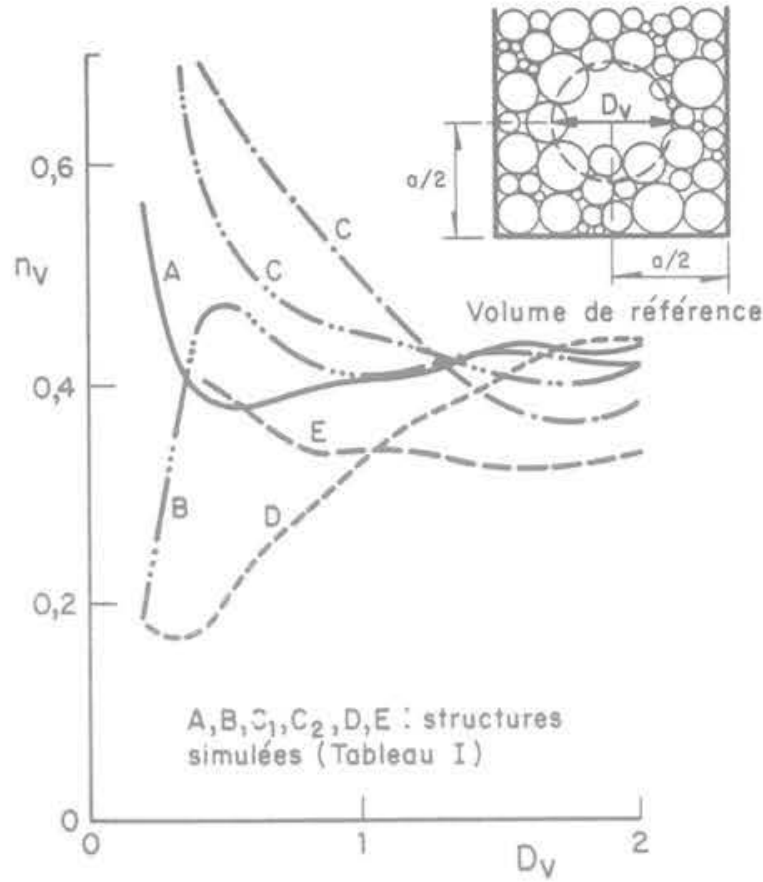

Fig. 4 Porosité dans des volumes de diamètre variable

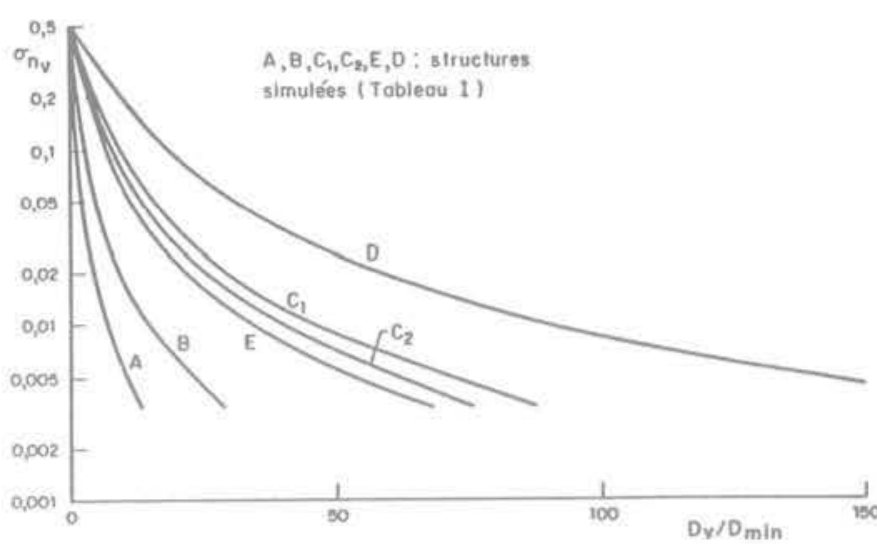

Fig. 5 Ecart-type de la porosité (en fonction de $D_{\gamma} / D_{\text {min }}$ )

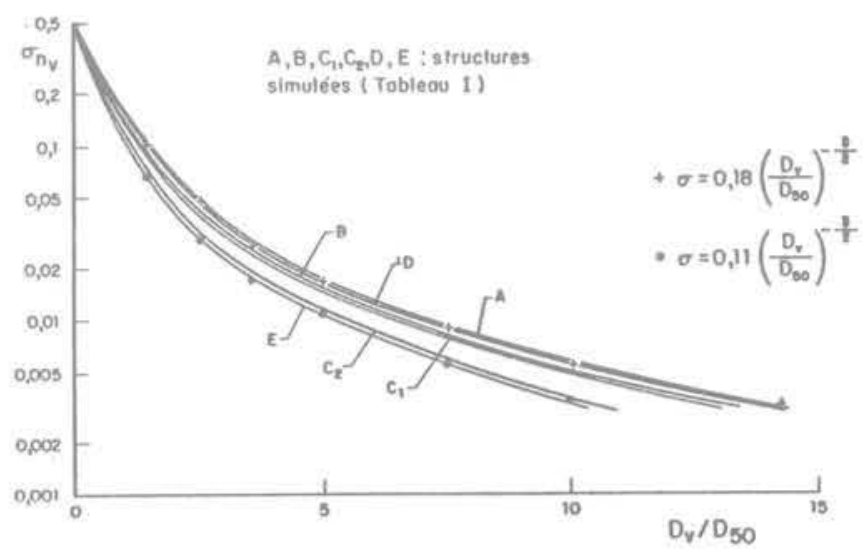

Fig. 6 Ecart-type de la porosité (en fonction de $D_{v} / D_{50}$ ) 


\subsection{Indice des vides}

L'indice des vides, paramètre plus usuel, en mécanique des sols, que la porosité, est lié à celle-ci par la relation :

$$
e=\frac{n}{1-n}
$$

Pour un volume donné $v_{t}$ l'indice des vides $e_{y}$ est lui aussi une variable aléatoire, dont l'espérance et la variance peuvent être estimées à partir de celles de $\mathrm{n}_{\mathrm{v}}$ par la méthode des moments de premier ordre (Benjamin, 1970) :

$$
\begin{aligned}
& E\left\{e_{v}\right\}=\frac{n}{1-n}+\left.\frac{1}{2} \frac{d^{2} e_{v}}{d n_{v}^{2}}\right|_{m} \operatorname{var}\left[n_{v}\right] \\
& E\left\{e_{v}\right\}=\frac{n}{1-n}+\frac{1}{(1-n)^{3}} v_{n_{v}}^{2} \\
& \operatorname{var}\left[e_{v}\right]=\left[\left.\frac{d e_{v}}{d n_{v}}\right|_{m}\right]^{2} \operatorname{var}\left[n_{v}\right] \\
& \operatorname{var}\left[e_{v}\right]=\sigma_{\theta_{v}}^{2} \approx \frac{1}{(1-n)^{4}} \sigma_{n_{v} .}^{2} .
\end{aligned}
$$

On voit que les mesures de l'indice des vides effectuées dans de petits échantillons tendent à surestimer systématiquement la valeur réelle de cette propriété. Par ailleurs, le coefficient de variation de l'indice des vides est supérieur à celui de la porosité puisque :

$$
\frac{\sigma_{e_{v}}}{e}=\frac{1}{(1-n)} \frac{\sigma_{n_{v}}}{n}
$$

L'erreur relative sur l'indice des vides est donc toujours supérieure, de 1,5 à 2 fois, à celle qui affecte la porosité. Ces résultats montrent que l'indice des vides est un paramètres plus sensible que la porosité à l'effet d'èchelle géométrique.

\subsection{Poids volumique}

Le poids volumique sec $y_{d}$ d'un sol s'exprime en fonction de la porosité comme suit:

$$
\gamma_{d}=\gamma_{s}(1-n)
$$

où $\gamma_{s}$ est le poids volumique des solides.

Si l'on admet que le poids volumique des solides est une constante, on a simplement, pour un volume $v$ :

$$
\begin{aligned}
& E\left\{\gamma_{d v}\right\}=\gamma_{s}(1-n)=\gamma_{d} \\
& \operatorname{var}\left[\gamma_{d v}\right]=\sigma_{\gamma_{d v}}^{2}=\gamma_{s}^{2} \sigma_{n_{v}}^{2}
\end{aligned}
$$

et par ailleurs :

$$
\frac{\sigma_{\gamma_{d v}}}{\gamma_{d}}=\frac{n}{1-n} \frac{\sigma_{n v}}{n} \text {. }
$$

L'erreur relative sur le poids volumique due à l'effet d'échelle est donc généralement légèrement inférieure à celle qui affecte la porosité.

\subsection{Granulométrie}

Le problème de la variation spatiale des pourcentages granulométriques peut être abordé d'une façon analogue à celui de la porosité. II suffit de définir une famille de fonctions aléatoires $K_{0}(\bar{x})$, telles que :

$K_{D}(\bar{x})=1$ si $\bar{x}$ est dans un pore ou dans un grain de diamètre plus petit que $D$;

$K_{D}(\bar{x})=0$ si $\bar{x}$ est dans un grain de diamètre plus grand que $D$
On a :

$$
\begin{aligned}
E\left\{K_{D}(\bar{x})\right\} & =G(D) \\
\operatorname{var}\left[K_{D}(\bar{x})\right] & =G(D)[1-G(D)]
\end{aligned}
$$

où $G(D)$ est la porosité obtenue en assimilant à des pores les particules de diamètre inférieur à $D$, avec en particulier $\mathrm{G}(0)=\mathrm{n}$.

La granulométrie usuelle $F(D)$ (pourcentage en volume de particules de diamètre inférieur à D) s'écrit :

$$
F(D)=\frac{G(D)-G(0)}{1-G(0)}
$$

Dans un volume $v$, on définit les variables aléatoires $G_{v}(D)$ et $F_{v}(D)$.

L'espérance de $G_{v}(D)$ et sa variance peuvent être estimées pour chacune des structures simulées en utilisant la méthode décrite plus haut pour la porosité. Les paramètres de $F_{\mathrm{v}}(\mathrm{D})$ s'en déduisent par la méthode des moments de premier ordre:

$$
\begin{aligned}
& E\left\{F_{v}(D)\right\} \approx F(D) \\
& \quad-\frac{1-G(D)}{(1-n)^{3}} \operatorname{var}\left[G_{v}(0)\right] \\
& +\frac{1}{(1-n)^{2}} \operatorname{cov}\left[G_{v}(d), G_{v}(0)\right] \\
& \operatorname{var}\left[F_{v}(D)\right]=\sigma_{F_{v}}^{2}(D) \approx \frac{1}{(1-n)^{2}} \operatorname{var}\left[G_{v}(D)\right]+\left[\frac{1-G(D)}{(1-n)^{2}}\right]^{2} \\
& \operatorname{var}\left[G_{v}(0)\right]-\frac{1-G(D)}{(1-n)^{3}} \operatorname{cov}\left[G_{v}(D), G_{v}(0)\right]
\end{aligned}
$$

La covariance de $G_{v}(D)$ et $G_{v}(0)$ peut être évaluée à l'aide d'un estimateur analogue à celui de l'équation 7.

L'écart-type $\sigma_{F_{v}}(D)$ a été calculé pour plusieurs valeurs de $D$ à l'aide de l'équation 27 et pour chacune des structures simulées non uniformes. Le coefficient de variation $\sigma_{F_{v}}(D) / F_{v}(D)$ obtenu s'est avéré peu sensible à la valeur de D. Sur la figure 7 on a représenté la variation de sa valeur moyenne en fonction des dimensions de l'échantillon. Pour un même rapport $D_{v} / D_{50}$, le coefficient de variation de $F_{v}(D)$ est plus petit pour les matériaux à granulométrie variée que pour les matériaux à granulométrie serrée. Par ailleurs, il est à nouveau possible d'ajuster aux courbes obtenues des expressions du type :

$$
\frac{\sigma_{F_{v}}(D)}{F_{v}(D)}=k\left(D_{v} / D_{50}\right)^{-3 / 2}
$$

où $\mathrm{k}$ est de l'ordre de 1,3 pour les matériaux les plus uniformes et 0,75 pour les matériaux à granulométrie étalée.

\section{Applications}

Les résultats obtenus permettent d'évaluer l'effet d'échelle en termes probabilistes. On peut en particulier :

- définir des intervalles de confiance pour les caractéristiques physiques d'une masse granulaire à partir de mesures effectuées sur de petits èchantillons;

- définir les dimensions minima d'un échantillon requises pour obtenir ces propriétés avec un certain degré de confiabilité;

- tester l'hypothèse d'homogénéité d'un milieu granulaire à partir des caractéristiques de plusieurs échantillons. 


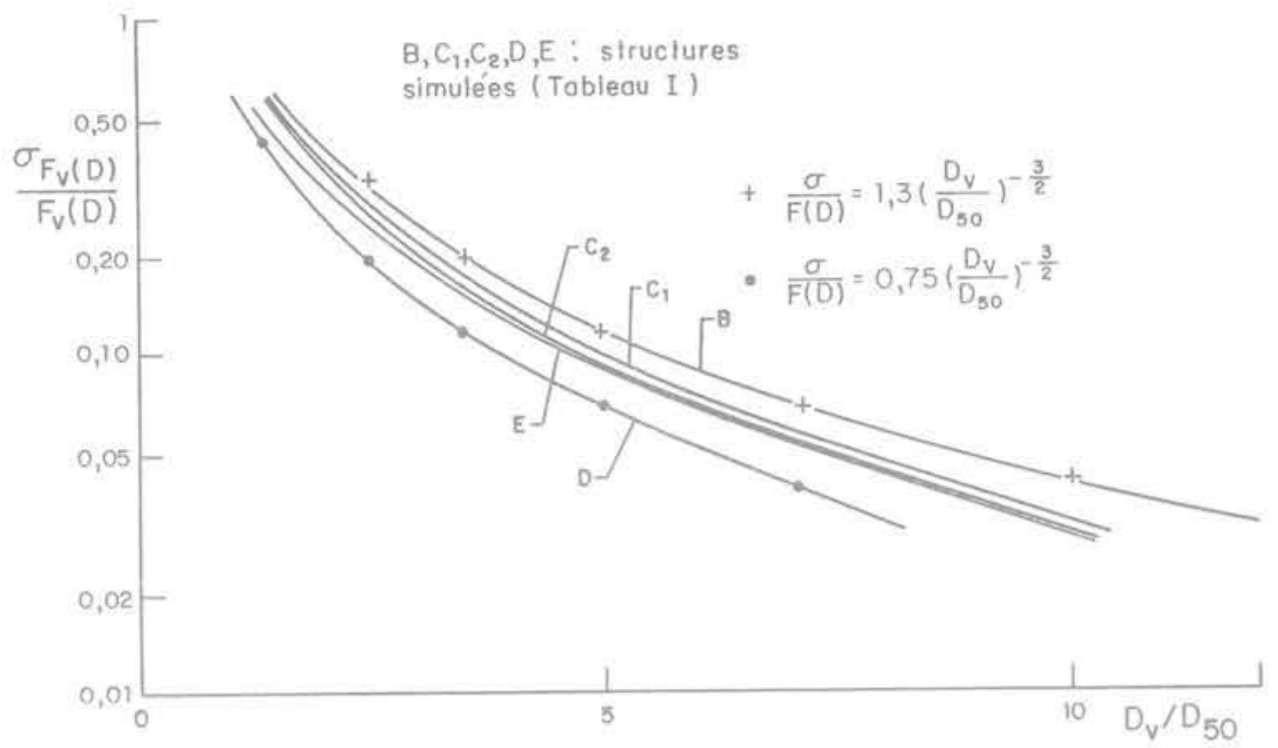

Fig. 7 Coefficient de variation des pourcentages granulométriques

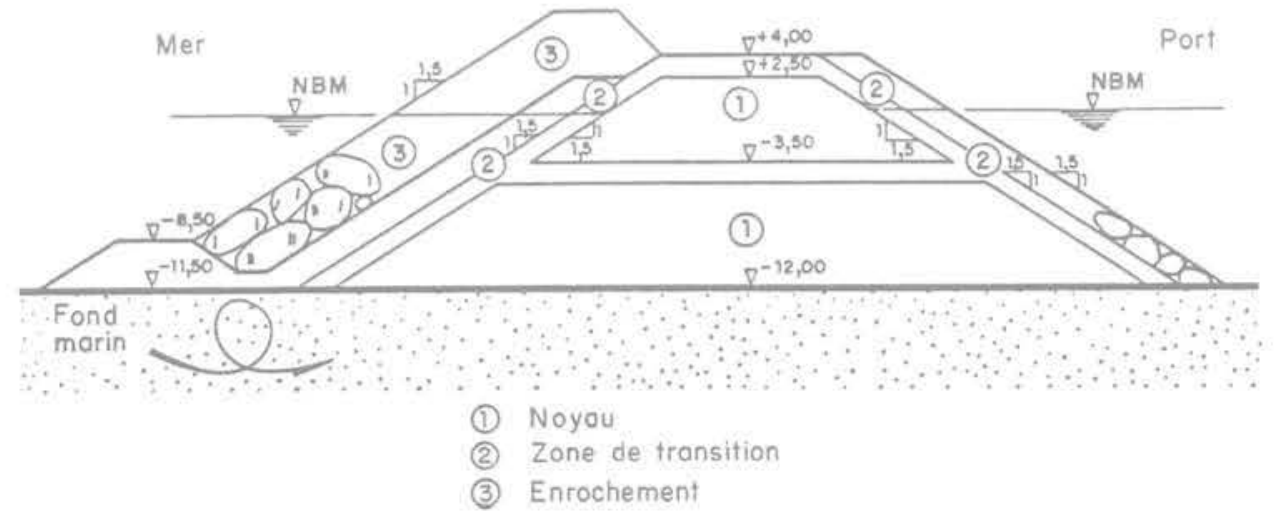

Fig. 8 Digue du port de El Ostion

C'est sans doute l'aspect des dimensions minima d'un échantillon qui présente le plus d'intérêt pratique, en particulier pour le contrôle des ouvrages en terre, ainsi que pour les essais mécaniques en place et en laboratoire.

\subsection{Contrôle des ouvrages en terre}

Les contrôles de poids volumique en place et de granulométrie sont nécessaires pour la construction des ouvrages en terre (barrages, digues, remblais). Lorsque le sol contient de gros éléments, l'effet d'échelle peut donner lieu à des erreurs importantes si l'échantillon analysé est trop petit. Pour obtenir le poids volumique avec une précision donnée, on peut définir les dimensions minima de l'échantillon à partir de l'équation 11. En effet, si l'on admet que la loi de probabilité de la porosité est sensiblement normale, l'erreur due à l'effet d'échelle est inférieure à $2 \sigma_{n v}$ avec un degré de certitude de $95 \%$.

Ainsi, pour que l'erreur sur la porosité soit inférieure par exemple à 0,01 avec une probabilité de $95 \%$, on devra avoir :

$$
D_{v} / D_{50}>(0,005 / k)^{-2 / 3}
$$

soit, pour les matériaux les moins denses:

$$
D_{v} / D_{50}>10,9 \text {. }
$$

Une erreur de 0,01 sur la porosité correspond à une erreur de 0,03 à 0,04 sur l'indice des vides et de $260 \mathrm{~N} / \mathrm{m}^{3}$ sur le poids volumique.

L'équation 30 a été appliquée aux matériaux de construction de la digue du port maritime de El Ostión, actuellement en construction au Mexique (fig. 8). Les résultats sont présentés sur le Tableau III. On voit que, même pour le noyau, l'effet d'échelle est très fort et que, dans la pratique, on pourra difficilement vérifier la compacité avec une précision satisfaisante. II s'agit là, bien sûr, d'un cas extrême: toutefois, même pour des sols moins grossiers, l'effet d'échelle peut prendre une certaine importance, en particulier si la méthode de contrôle porte sur un échantillon de volume réduit (méthode nucléaire par exemple).

Tableau III

Volume minimum des échantillons. Digue de El Ostión (Mexique)

\begin{tabular}{lrrr}
\hline Matériau (fig. 8) & $\begin{array}{c}D_{50} \\
m\end{array}$ & \multicolumn{1}{c}{$\begin{array}{c}D_{v} \\
m\end{array}$} & $\begin{array}{c}\text { Volume } \\
m^{3}\end{array}$ \\
\hline 1 (noyau) & 0,52 & 5,70 & 97 \\
2 (transition) & 1,25 & 13,60 & 1317 \\
3 (enrochement) & 2,45 & 26,70 & 9966 \\
4 (blocs de béton) & 2,60 & 28,30 & 11867 \\
\hline
\end{tabular}




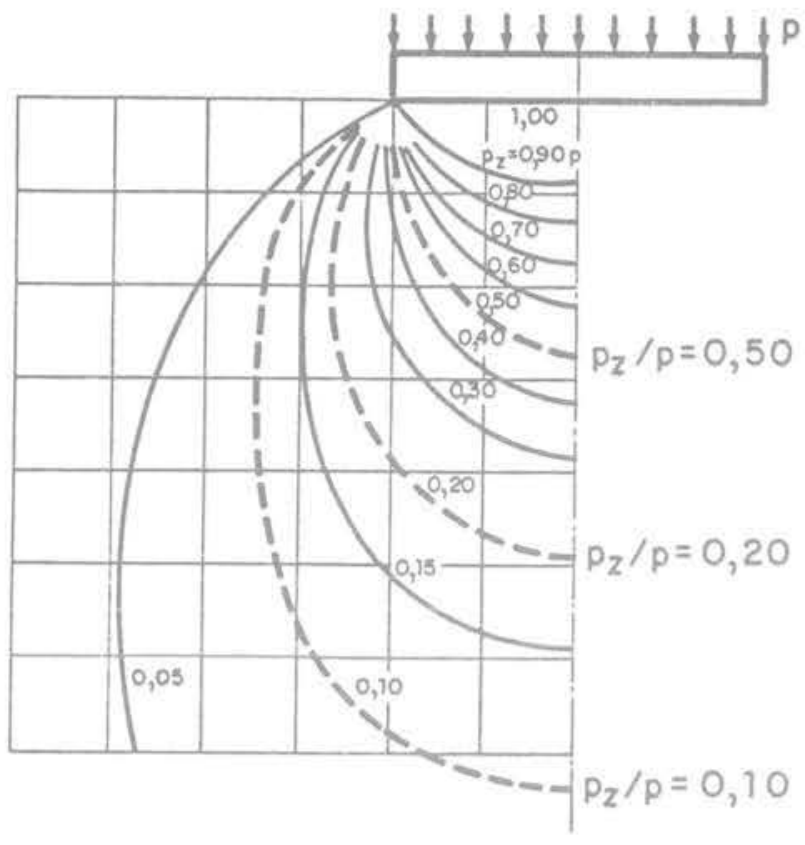

Fig. 9 Essai à la plaque

\subsection{Essais en place}

Les résultats des essais mécaniques en place sur des matériaux granulaires peuvent être faussés par l'effet d'échelle. Ceci est clair, par exemple, dans le cas de l'essai à la plaque. Dans cet essai, pour que la porosité et la granulométrie de la zone soumise au chargement soient représentatives de celles du milieu, on doit tenir compte des dimensions relatives des particules et des bulbes de contraintes verticales $p_{2}$ (fig. 9). Si l'on applique, par exemple, la formule 30 au bulbe correspondant à $p_{2} / p=0,50, p$ étant la pression appliquée, on trouve que le diamètre de la plaque doit être supérieur à vingt fois le $D_{50}$ du matériau étudié.

\section{rappel figure 9}

Des considérations analogues pourraient être faites pour d'autres essais en place tels que l'essai pressiométrique, les essais de pénétration, etc.

\subsection{Essais de laboratoire}

Le choix des dimensions des échantillons représentatifs intacts ou reconstitués à soumettre à des essais mécaniques en laboratoire doit tenir compte de l'effet d'échelle. Pour les échantillons intacts, la représentativité de la porosité et de la granulométrie peut être évaluée à laide des équations 11 et 28. Dans le cas des échantillons reconstitués, le problème se pose généralement de la même façon pour la granulométrie, mais il n'existe plus de relation statistique entre la porosité de l'échantillon et celle du milieu.

Diverses techniques ont été développées pour vérifier I'homogénéité des échantillons. Ces techniques, destructives ou non, consistent à déterminer la porosité dans diverses zones des échantillons. Ce type de contrôle peut être sensible à l'effet d'échelle si les zones étudiées ont des dimensions trop faibles par rapport à celles des grains.

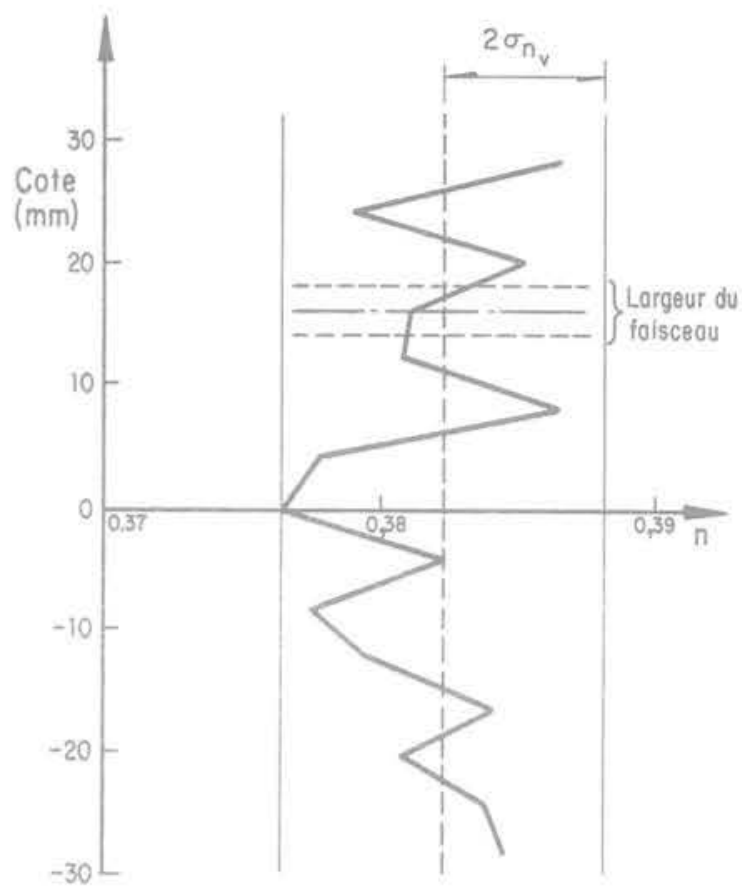

Fig. 10 Contrôle gammamétrique d'homogénéité

A titre d'exemple, on a représenté sur la figure 10 les résultats obtenus par étude gammamétrique d'un échantillon de sable $\left(D_{50}=1,3 \mathrm{~mm}, C_{u}=1,4\right)$. La méthode consiste à mesurer à différentes hauteurs l'absorption d'un faisceau de rayons Gamma de $4 \mathrm{~mm}$ de diamètre traversant l'échantillon sur une longueur de $70 \mathrm{~mm}$ (Bouvard, 1982). D'après l'équation 10, la dispersion par effet d'échelle $\left(2 \sigma_{n v}\right)$ est, pour ce volume, de l'ordre de 0,005 à 0,008 suivant l'état de compacité du matériau. Les variations présentées sur la figure 10 ne sont donc pas significatives; l'hypothèse d'homogénéité ne doit pas être écartée car ces variations peuvent être simplement dues à l'effet d'échelle.

\subsection{Effet d'échelle géométrique et propriétés mécaniques}

II est bien connu que la résistance au cisaillement des sols déterminée au laboratoire est sensible à la relation $D_{c} / D_{\max }$ ou $D_{c}$ est la dimension de l'échantillon et $D_{\max }$ le diamètre maximum des particules. Ainsi, l'angle d'inclinaison $\phi_{0}$ d'une droite tangente au cercle de Mohr de rupture et passant par l'origine varie en fonction de $D_{c} / D_{\max }$ comme l'indique la figure 11 (Marsal, 1969). II semble que $\phi_{0}$ n'atteigne une valeur sensiblement constante que pour des valeurs de $D_{c} / D_{m a c}$ supérieures à 10 . Ce résultat est à rapprocher de ceux obtenus plus haut (éq. 30). Les effets d'échelle géométrique et mécanique semblent donc še présenter à peu près simultanément. Ils ne doivent toutefois pas être confondus puisque le premier est de nature statistique alors que le second est lié à l'apparition de phénomènes mal connus comme laugmentation de la dilatance et de l'importance relative des restrictions aux frontières de l'échantillon. 


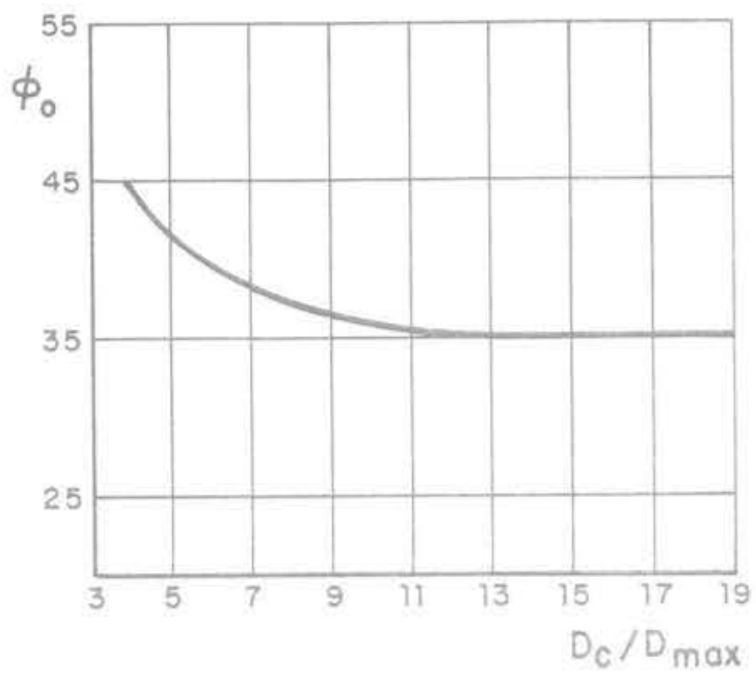

Fig. 11 Effet d'échelle mécanique

\section{Conclusion}

L'effet d'échelle géométrique dans les milieux granulaires a pu être défini en termes probabilistes et évalué numériquement au moyen de la théorie des fonctions aléatoires et d'un modèle qui permet de simuler la structure de ces milieux. On a pu obtenir certains résultats généraux et définir des ordres de grandeur dont on devra tenir compte dans la pratique. Ces élèments pourront également être utiles pour une étude rationnelle de l'hétérogénéité des sols dans le cas où ce phénomène contribue, en même temps que l'effet d'échelle, à la dispersion de leurs caractéristiques physiques.

\section{Notations}

a

$C\left(\vec{x}_{1}, \bar{x}_{2}\right)$

$\mathrm{C}_{\mathrm{u}}$ $\operatorname{cov}[$ ]

$\mathrm{D}_{\mathrm{c}}$

$D_{\max }$

$D_{\min }$
Arête du moule.

Autocovariance de la fonction aléatoire $K(\bar{x})$.

Coefficient d'uniformité.

Covariance.

Diamètre d'un échantillon.

Diamètre maximum des grains.

Diamètre minimum des grains.

\section{Références bibliographiques}

MATHERON G. (1967), Chap. V. Genèse de la loi de Darcy. Éléments pour une théorie des milieux poreux,

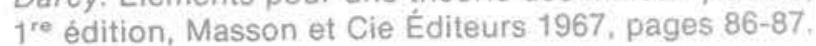

PERALTA-FABI R. et HERNANDEZ G. (1982). Procesamiento digital de imágenes de microscopia electrónica de barrido, Octubre 1982. Memoria II Simposio de Instrumentación, Oaxtepec, México.

AUVINET G. (1977). Structure des milieux pulvérulents, Juillet 1977, comptes rendus du IX Congrès International de Mécanique des Sols et des Travaux de Fondations, Tokyo.
$D_{10}, D_{50}, D_{60}$

$D_{v}$

$e_{1} e_{v}$

E \{\}

$F(D), F_{v}(D)$

$G(D), G_{v}(D)$

h

i

$K\left(\bar{x}, K_{D}(\bar{x})\right.$

k

L

lo

$n, n_{v}$

N

p. $p_{z}$

$R\left(\bar{x}_{1}, \bar{x}_{2}\right)$

$R_{\text {L. }} T$

v

var[]

$\bar{x}$

$\gamma_{d}, \gamma_{a v}$

$\gamma_{\mathrm{s}}$

$\rho\left(\bar{x}_{T}, x_{2}\right)$

$\sigma_{y}^{2}$

$\sigma_{y}$

$\phi_{0}$

$\left.\frac{d e_{v}}{d n_{v}}\right|_{m}$
Diamètres correspondant à des tamisats de 10,50 et $60 \%$.

Diamètre d'un volume sphérique de référence $v$.

Indices des vides en masse indéfinie et dans un volume $v$.

Espérance mathématique.

Granulométries, en masse indéfinie et dans un volume $v$.

Porosités virtuelles obtenues en assimilant à des vides les grains de diamètre inférieur à $D_{1}$ en masse indéfinie et dans un volume $v$.

Distance entre deux points du milieu. Vecteur unitaire.

Fonctions aléatoires spatiales.

Coefficient empirique.

Longueur des segments d'estimation. Distance aux parois.

Porosités en masse indéfinie et dans un volume $\mathrm{v}$.

Nombre d'échantillons.

Pression verticale en surface et à la profondeur $z$.

Autocorrélation de la fonction aléatoire $\mathrm{K}(\overline{\mathrm{x}})$.

Estimateur de $R\left(\bar{x}_{1}, \bar{x}_{2}\right)$.

Volume de référence.

Variance.

Point du milieu.

Poids volumique sec en masse indéfinie et dans un volume $v$.

Poids volumique des solides.

Coefficient d'autocorrélation.

Variance de la variable aléatoire y. Écart-type de la variable aléatoire $y$. Angle de frottement.

Dérivée de la variable aléatoire e e par rapport à $n_{v}$, calculée pour la valeur moyenne de $e_{\mathrm{v}}$

BENJAMIN J. et CORNELL C.A. (1970). Chap. II.4 Moments and expectation, Probability, Statistics and Decision for Civil Engineers, $1^{\text {re }}$ édition, McGraw-Hill, Éditeur 1970, pages 180-188.

BOUVARD D. (1982). Rhéologie des milieux pulvérulents: Étude expérimentale et identification d'un loi de comportement. Thèse présentée à I'Université de Grenoble, soutenue le 22 janvier 1982, pour obtenir le grade de Docteur-Ingénieur.

MARSAL R. (1969). Minutes of Specialty Session $n^{\circ}$ 13, August 1969, Proceedings Specialty Session $n^{\circ} 13$, Seventh International Conference on Soil Mechanics and Foundations Engineering. Publicación $n^{\circ}$ E1, Instituto de Ingeniería, UNAM, México. 